\title{
The influence of three endodontic sealers on bone healing: an experimental study
}

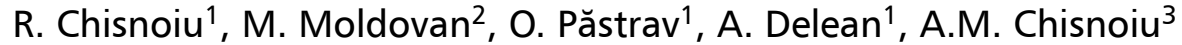 \\ 'Department of Odontology, "Iuliu Hațieganu" University of Medicine and Pharmacy, Cluj-Napoca, Romania \\ 2"Raluca Ripan" Institute for Research in Chemistry, "Babeș Bolyai" University, Cluj-Napoca, Romania \\ ${ }^{3}$ Department of Prosthodontics, "Iuliu Hațieganu" University of Medicine and Pharmacy, Cluj-Napoca, Romania
}

[Received: 31 March 2015; Accepted: 29 June 2015]

Background: The aim of this experimental study is to assess the bone healing phenomenon produced in the presence of 3 dental materials used for the root canal obturation.

Materials and methods: The biocompatibility of 3 endodontic sealers (a self-curing epoxy resin - AH Plus, a dual cure urethane dimethacrylate resin - RealSeal and a new dual cure endodontic hydroxyapatite based filling material) was investigated after intra-osseous implantation of the materials in rats' calvaria. Tissue reaction was studied at 2, 4, 6, 8, 10 and 12 weeks after implantation using calibrated image retrieval by Olympus. We took into consideration the presence of inflammatory cells (polymorphonuclear leukocytes, macrophages, plasma cells, lymphocytes and giant cells) and classified the aspects of the histological samples according to the following scale: 0 - no inflammation, 1 - mild, isolated inflammation, 2 - moderate, localised inflammatory reaction, 3 - severe, diffuse and intense inflammatory reaction.

Results: The inflammatory reaction was present at the 6 intervals for all the tested materials, but a decrease of the inflammatory infiltrate, statistically significant, until extinction for all tested materials was observed at the end of the experimental period. The reaction of bone tissue recovery was most intense in the case of the control area. Lower intensity osteogenesis phenomenon was observed in case of all 3 tested sealers at the end of the experimental period.

Conclusions: Biocompatibility and bone healing induction of the new hydroxyapatite based endodontic filling material is comparable to other commercial materials, AH Plus and RealSeal. (Folia Morphol 2016; 75, 1: 14-20)

Key words: root canal filling, bone tissue response, biocompatible materials, endodontic materials

\section{INTRODUCTION}

Endodontic materials used for root canal treatment consist of the basic filling material (gutta-percha) and the sealer. Due to the long-lasting direct contact between these materials and periapical tissues, their biocompatibility should be taken into consideration.
Biocompatibility describes the ability of a material to trigger an appropriate host response in a specific application. A biocompatible material cannot be completely inert. In fact, the adequacy of the host response is decisive. This characteristic is generally assessed by experts in comparison to products that are already on the market [1]. 
Grossman outlined some of the ideal characteristics for endodontic sealers: they should have efficient sealing capacity, they should be bacteriostatic and non-irritating to the periapical tissues, they should be soluble in a solvent but they should not disintegrate when in contact with tissue fluids, they should provide adequate working time for manipulation and placement into the root canal [5].

The development of new root canal filling materials, similar to dentin, based on calcium hydroxide and biodegradable polymer matrices, seemed promising in creating new endodontic materials.

Other types of bioactive polymer materials for use in dentistry are those based on amorphous calcium phosphate. The interest in these types of materials has increased among researchers due to their remineralisation effects on enamel, dentin and bone, and to their biocompatibility.

In aqueous media, amorphous calcium phosphate is transformed into hydroxyapatite, the major component of tooth enamel and bone mineral. In addition, amorphous calcium phosphate composites might be used as root canal sealers or as support for other types of dental restorations, with applications in both endodontics and periodontics, where such composites enable the development of healthy bones [6].

Considering the structure of the bone as a composite, hydroxyapatite is the mineral phase dispersed throughout a collagen matrix. In teeth and bones, calcium deficient hydroxyapatite is different from synthetic hydroxyapatite due to the substituents in its structure. Synthetic hydroxyapatite is an osteoconductive compound that chemically binds to enamel and dentin. Moreover, it has a large specific surface area, high chemical homogeneity and microstructural uniformity. It is also the least expensive of all the filling materials on the market [7].

The aim of this experimental animal study was to assess the bone healing phenomenon produced in the presence of several endodontic sealers: epoxy resin, urethane dimethacrylate resin and a new hydroxyapatite-based dental resin.

\section{MATERIALS AND METHODS}

Two conventional endodontic sealers were used in this study: self-cure AH Plus epoxy resin (Dentsply DeTrey GmbH, Konstanz, Germany) and dual-cure (photo-activation and auto polymerisation) RealSeal urethane dimethacrylate resin (SybronEndo, Orange,
CA, USA), together with a new dual-cure root canal filling material based on hydroxyapatite (NEM), developed in collaboration with Raluca Ripan Institute for Research in Chemistry, Cluj-Napoca, Romania. The chemical composition of the new root canal filling material was: organic phase: $33 \%$ Bis-GMA, $5 \% \mathrm{PCL}$, 34\% TEGDMA, 28\% UDMA, and inorganic phase: 10\% Zinc coloured glass, 30\% Hydroxyapatite-Zinc, 10\% Hydroxyapatite-Titanium, and radiopaque agents: $40 \%$ Zirconium oxide, $10 \%$ Ytterbium fluoride.

The animals used in this study were adult male Wistar rats weighing 180-200 g. The study was reviewed and approved by the Ethics Committee of our University. All ethical principles were respected. The animals were housed in an air-conditioned room $\left(21-23^{\circ} \mathrm{C}\right)$, maintained on a balanced diet and given water ad libitum.

\section{Procedure}

Experimental studies were conducted on 48 rats. In preparation for the experimental procedure, the animals were anesthetised with an intramuscular injection of Ketamine hydrochloride $(0.1 \mathrm{mg} / \mathrm{mL}) \mathrm{com}$ bined with Xylazine hydrochloride $(0.05 \mathrm{mg} / \mathrm{mL})$. After anaesthesia, a linear scalp incision was performed using number $15 \mathrm{~T}$ scalpel blade, the sides of the incision were stretched open and 4 osteotomies $(2 \mathrm{~mm}$ in diameter and $1 \mathrm{~mm}$ in depth) were performed using an inverted cone bur, $2.0 \mathrm{~mm}$ in diameter and $1.0 \mathrm{~mm}$ in length, under permanent saline solution cooling $[9,10]$. Paramedian osteotomies were performed in 2 horizontal and 2 vertical lines, $2 \mathrm{~mm}$ from each other. The first orifice was left empty (control orifice) and the other 3 were filled with one of the root canal sealers: AH Plus, RealSeal and NEM.

All materials were prepared according to the manufacturer's specifications. After irrigation with sterile saline solution, the materials were placed into the prepared osteotomies. The incision was sanitized and $12 \%$ oxytetracycline powder was applied over the incision, further being sutured with separate silk stitches (number 4.0). The rats were monitored regularly for signs of infection.

Four rats in each group were sacrificed every 2 weeks, starting with the end of the second week after surgery. The last 4 rats in each group were euthanized in the 12th week using a lethal dose of Ketamine Hydrochloride. The calvaria containing the 4 orifices were harvested from each rat. 


\section{Histological analysis}

The samples were fixed by immersion in $4 \%$ chemically buffered formaldehyde for $48 \mathrm{~h}$. Sample decalcification was achieved by treating the fixed specimens with $8 \% 1 / 1$ diluted formic acid/hydrochloric acid for 3 weeks. Further, samples were technically prepared for paraffin inclusion. This method consists of a few standard steps: washing, dehydration in $70 \%, 80 \%$, $90 \%$ alcohol, followed by inclusion in paraffin at $62^{\circ} \mathrm{C}$ under vacuum for $6 \mathrm{~h}$, using a high melt point wax.

Following paraffin inclusion, the samples were sectioned to $4 \mu \mathrm{m}$ in thickness using a Leica microtome and then they were subjected to histological sampling. Sectioning was performed perpendicular to each hole to observe any possible changes.

The following steps consisted in sample dewaxing using $100 \%$ xylene, hydration in $100 \%, 96 \%$ and $70 \%$ alcohol and staining. Haematoxylin and eosin staining (H\&E) was used to distinguish different cell types.

Histological samples were examined and photographed using an Olympus BX 51 image calibration and retrieval microscope. Image acquisition and processing was done using Olympus Cell B image analysis software.

Each sample was carefully examined to observe bone resorption, inflammatory infiltrates, bone remodelling and bone formation.

Representative sections were identified and photographed using $4 \times, 10 \times, 20 \times$ and $40 \times$ objectives. Performed according to standard criteria, the histological examination focused on inflammation around the area where the root filling materials were implanted, specifically at the contact with the existing bone, or at the edge of the cavity for controls $[9,10]$.

The presence of inflammatory cells (polymorphonuclear, leukocytes, macrophages, plasma cells, lymphocytes and giant cells) was taken into consideration and the histological aspects of the samples were classified using a grading scale [16] as follows: 0 - no inflammation, 1 - mild, isolated inflammation (few macrophages), 2 -moderate, localised inflammation (many macrophages), 3- severe, diffuse and intense inflammation (abundant macrophages).

The normal distribution of the samples was tested using the Wilcoxon signed-rank test. After parametric distribution, the differences in inflammatory indexes between the controls and experimental groups, on the one hand, and the 3 materials, on the other hand, as well as those between the groups treated with conventional materials and the ones treated with the new material based on hydroxyapatite, were analysed using the $\chi^{2}$ test. The statistical significance was set at a $p$ value of $<0.05$. Statistical analysis was performed using MedCalc version 15.2.1 software (MedCalc Software bvba, Ostend, Belgium).

\section{RESULTS}

The variation in the inflammatory response was generally similar for all the study groups, ranging from high intensity for the first two intervals ( 2 and 4 weeks) to a gradual decrease and complete disappearance of all inflammatory processes and even new bone formation towards the end of the experiment. Statistical validation was performed for all the results (control: $p=0.005$, AH Plus: $p=0.003$, RealSeal: $p=0.023$, NEM: $p=0.005$ ).

Two weeks after implantation, an inflammatory reaction could be observed in all cases, with numerous macrophages and new fibrous connective tissue development around the implanted material. Ischaemic bone necrosis was also observed. In controls, the cavity was taken over by a new connective tissue (granulation tissue) and ischaemic bone necrosis was present as well (Fig. 1).

After 4 weeks, the degree of inflammation in the RealSeal group was $2(2 ; 2.75)$ and the histological examination revealed the development of granulation tissue around the material. The inflammatory infiltrate in the AH Plus group was lower (median $2.50[2 ; 3]$ ) than in the case of the other tested materials, higher in macrophages, while the NEM (median 2.50 [2; 3]) showed a granulomatous reaction around the material. The presence of both granulation tissue and osteoblasts was observed in controls (median 2 [2; 2.75]) (Fig. 2).

After 6 and 8 weeks, the RealSeal (median 1.50 $[1 ; 2.75]$ ) and AH Plus (median $1[1 ; 1.75]$ ) groups revealed the presence of a mature fibrous connective tissue with mild inflammatory infiltration, mainly consisting in macrophages. Productive osteoblasts were identified in bone ends. A mature, well-vascularised tissue was observed in the group treated with NEM (median $1[1 ; 1.75]$ ), with a moderate inflammatory reaction, consisting mainly of macrophages and few neutrophils. Fibrous connective tissue was identified in controls, accompanied by incipient osseous metaplasia, osteoblast proliferation and osteoid formation.

After 10 weeks and the end of the experimental period ( 12 weeks), there was an acute decrease in inflammatory infiltrates in the experimental groups, 

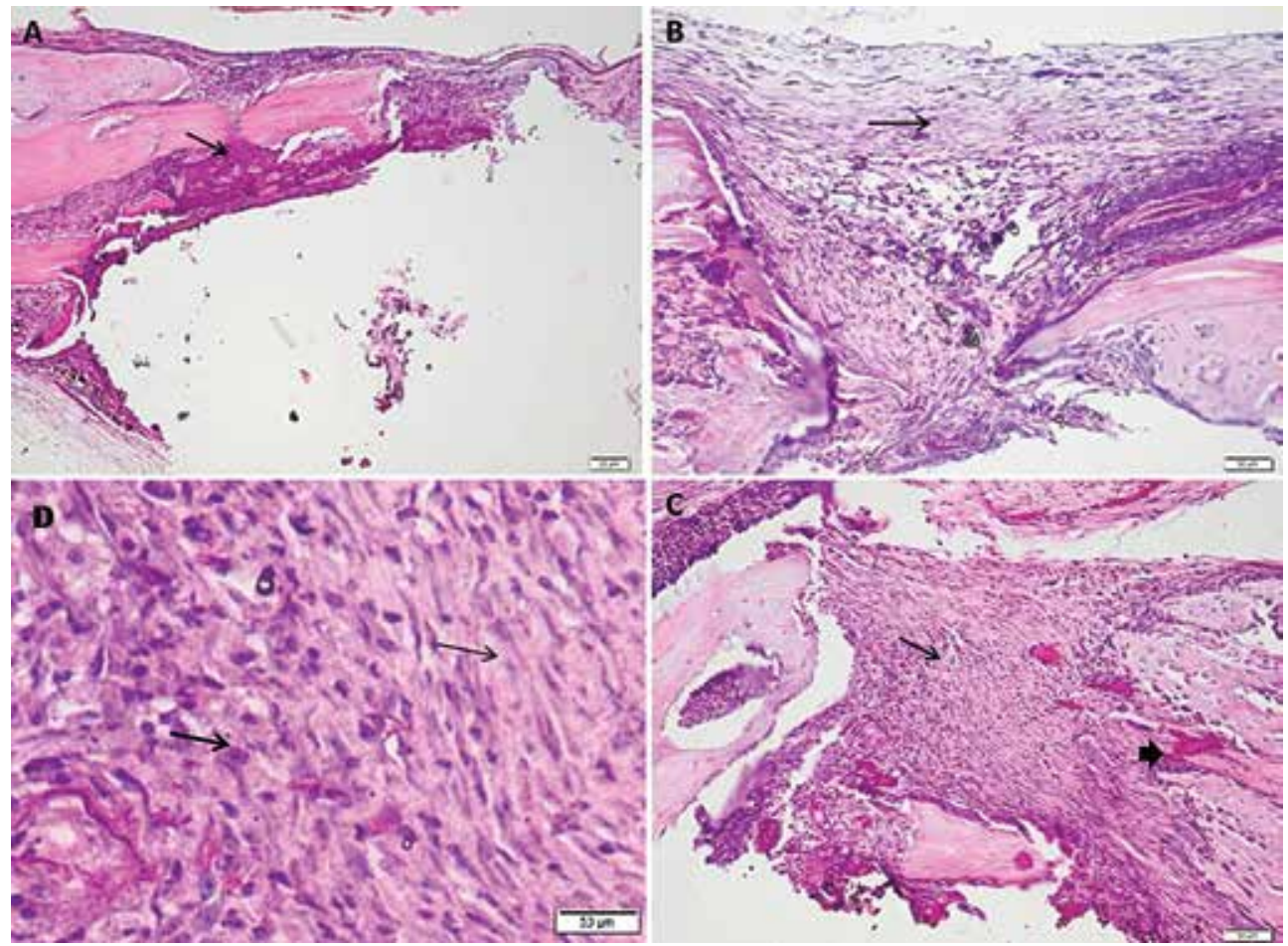

Figure 1. Comparative aspects at 2 weeks of experiment. Haematoxylin and eosin staining; $\mathbf{A}$. RealSeal. Granulation tissue around the implanted material (arrow); B. AH Plus. Moderate inflammatory infiltrate (arrow); C. Control. New highly vascularised connective tissue (thin arrow), ischaemic bone necrosis (thick arrow); D. NEM. Numerous inflammatory cells, mainly macrophages (thick arrow), proliferative connective tissue (thin arrow).
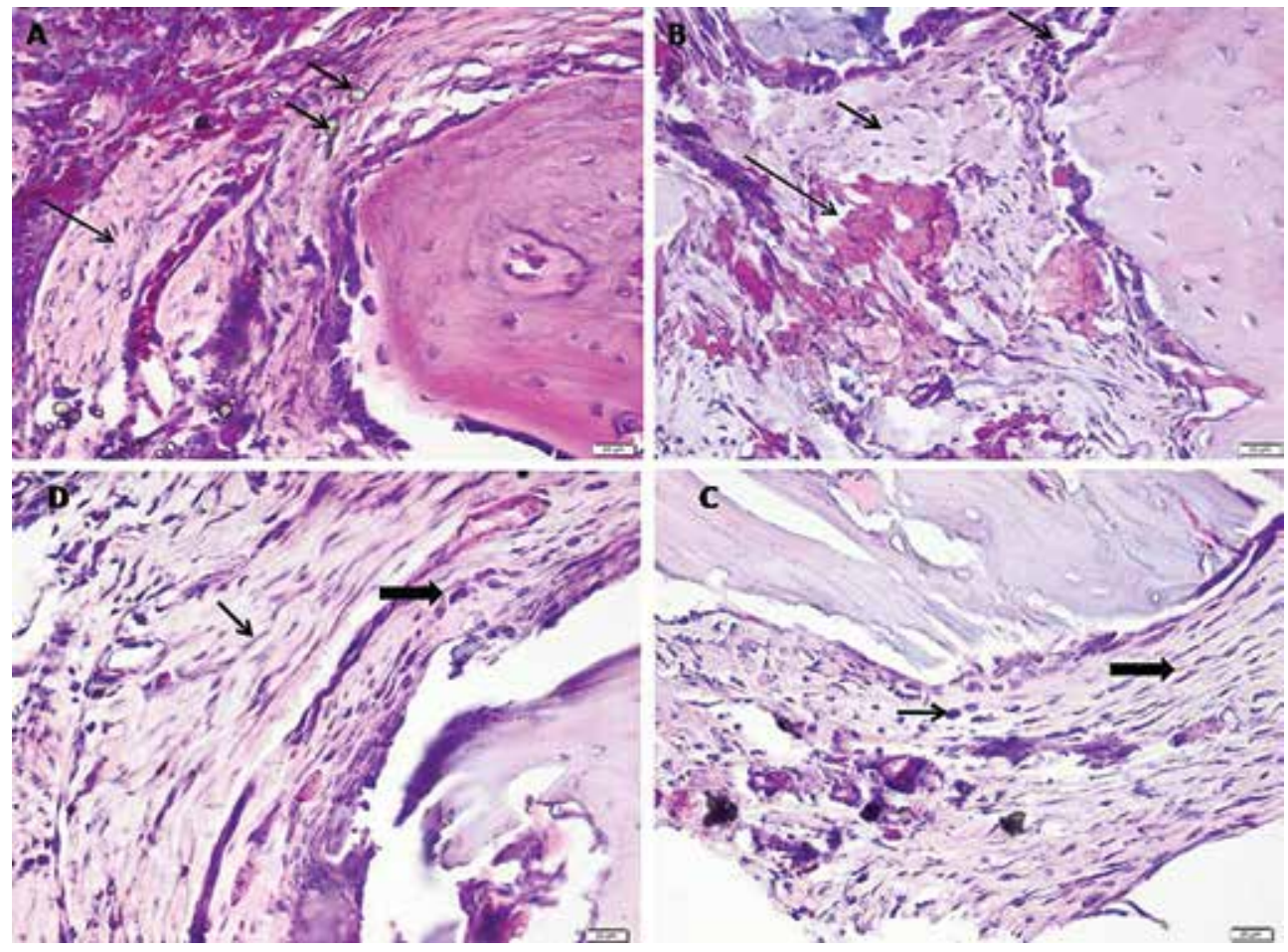

Figure 2. Comparative aspects at 4 weeks of experiment. Haematoxylin and eosin staining; A. RealSeal. New fibrous connective tissue (thin arrow), material crystals (thick arrow); B. AH Plus. Fibrous connective tissue (short arrow), ischaemic bone necrosis (long arrow), osteoblast alignment (thick arrow); C. Control. Fibrous connective tissue (thin arrow), inflammatory cells (macrophages - thick arrow); D. NEM. Fibrous connective tissue (thick arrow), numerous macrophages (thin arrow). 

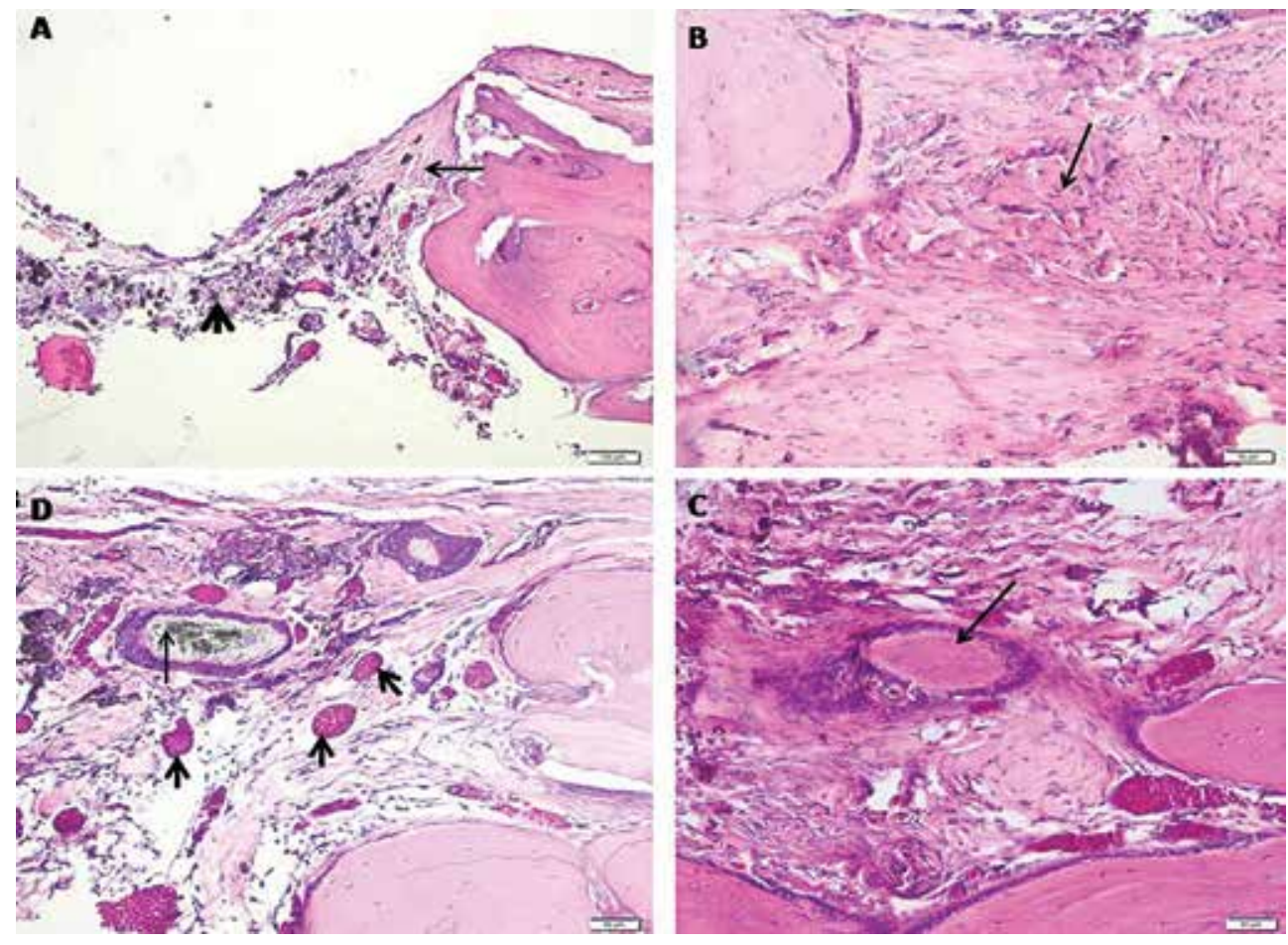

Figure 3. Comparative aspects at 12 weeks of experiment. Haematoxylin and eosin staining; A. RealSeal. Mature fibrous connective tissue (arrow), granulation tissue with frequent high calibre blood vessels (arrow tip); B. AH Plus. Osteoid matrix formation (arrow); C. Control. Osteoid matrix surrounded by osteoblasts (arrow); D. NEM. Mature connective tissue with blood vessels (thick arrow), material crystals surrounded by inflammatory cells (thin arrow).

Table 1. Statistical differences ( $p$ values) related to inflammatory indices between the materials studied and controls

\begin{tabular}{lcccccc}
\hline & $\mathbf{2}$ weeks & $\mathbf{4}$ weeks & $\mathbf{6}$ weeks & $\mathbf{8}$ weeks & 10 weeks & 12 weeks \\
\hline AH Plus & 0.317 & 0.127 & 0.127 & 0.186 & 0.186 & 0.495 \\
RealSeal & 0.096 & 0.317 & 0.127 & 0.098 & 0.040 & 0.495 \\
NEM & 0.186 & 0.127 & 0.127 & 0.186 & 0.040 & 0.186 \\
\hline
\end{tabular}

Table 2. Statistical differences ( $p$ values) related to inflammatory indices between the materials studied and NEM

\begin{tabular}{lcccccc}
\hline & $\mathbf{2}$ weeks & 4 weeks & $\mathbf{6}$ weeks & $\mathbf{8}$ weeks & $\mathbf{1 0}$ weeks & 12 weeks \\
\hline AH Plus & 0.317 & 1.000 & 1.000 & 1.000 & 0.317 & 0.495 \\
RealSeal & 0.495 & 0.495 & 1.000 & 0.405 & 0.317 & 0.495 \\
\hline
\end{tabular}

with the presence of mature well-vascularised connective tissue. Fibrous connective tissue metaplasia and osteoid matrix formation were noticed in the AH Plus group (median 0.50 [0;0.75]). The healing processes was obvious in controls (median 0 [0; 0.75]), with the formation of osteoid matrix and primary callous. The formation of connective capsule around the material was observed in only one section performed in the group treated with NEM (Fig. 3).

Inflammatory indices for the tested materials were assessed for each time interval and there were no statistically significant differences when compared with controls (Table 1). The changes observed in the case of the two conventional materials did not significantly differ from those observed NEM (Table 2).

\section{DISCUSSION}

As there is a well-documented model for the assessment of bone healing, experimental studies on the rat skull is the gold standard for testing tissue reaction to root canal filling material $[3,13,14]$. 
For each group, the control orifice was 1 of the 4 orifices made in the calvaria, which was free of any tested material. Therefore, each sample had its own control area, which we considered significant in our study. We compared the natural bone healing process with that occurring in the presence of the 3 materials tested for 6 different time intervals $(2,4$, $6,8,10,12$ weeks).

In a study comparing the intraosseous biocompatibility of Epiphany materials (endodontic sealer with the same chemical composition as RealSeal) and $\mathrm{AH}$ Plus materials for 4- and 12-week intervals, Sousa et al. [15] observed that, in the presence of AH Plus, the inflammatory reaction decreased from severe to moderate, while in the presence of Epiphany it was reduced or even became absent.

In a histopathological study on the subcutaneous tissue response to different endodontic sealers, Farhad et al. [4] found that the inflammatory reaction was moderate in the case of AH Plus 2 weeks after treatment, and reduced after 4 and 8 weeks. For Epiphany, the inflammatory reaction decreased from moderate, after 2 weeks, to reduced, after 8 weeks.

The results of the current study are comparable to those obtained in the above studies, observing a progressive, statistically significant decrease in inflammatory infiltrates, up to a complete absence, for all tested materials. Similar observations were made by Onay et al. [12] or Batista et al. [1], concluding that the two materials (AH Plus and Epiphany) are biocompatible.

On the other hand, Bouillaguet et al. [2] and Lodiene et al. [8] obtained different results in an in vitro study, demonstrating the severe cytotoxicity of Epiphany, probably due to the remaining monomer. The drawback of in vitro studies is that cytotoxicity is assessed shortly after mixing the endodontic sealer, without assessing its behaviour over time.

Dual polymerisation not only allows a uniform polymerisation, but also the removal or reduction in residual monomer. Diffuse scattering of monomer can cause inflammation and infection when in contact with the tissue, which is undesirable.

NEM was comparable to the other two materials tested, having similar behaviour and causing a pronounced inflammatory reaction in the first time interval, which decreased until the end of the experimental period. The presence of hydroxyapatite in the material did not produce the desired effect, no accelerated bone reconstruction being observed when compared with the other two materials. This could be explained by the fact that, although the mechanical properties of calcium phosphate can be improved by incorporation into polymer matrices, as done in our study (the new material based on hydroxyapatite), there are still a few disadvantages. Contraction during polymerisation and partial conversion of vinyl groups are the main drawbacks of polymer matrices [17]. Polymerisation shrinkage may cause poor adhesion and internal/external morphological defects, including fractures and cracks determined by tension inside the composite. Severe inflammatory reaction, occurring sporadically for RealSeal and NEM, is probably due to the residual monomer, which did not achieve polymerisation. Incomplete conversion of vinyl groups can cause the reduction in biocompatibility due to the possible infiltration of monomers.

In this study, tissue inflammatory response was assessed based on the amount of inflammatory cells using a grading scale. According to Olsson et al. [11], the quantitative assessment of the inflammatory response is possible when there is a significant difference between the groups. On the other hand, the qualitative assessment of the inflammatory response cannot accurately compare different materials or different time intervals for the same material. The lack of statistically significant differences between some groups of animals used in our study may be due to the quantitative method used.

\section{CONCLUSIONS}

The biocompatibility of NEM (dual-cure material) is comparable to other conventional endodontic materials, such as AH Plus (self-cure material, widespread among practitioners) and RealSeal (dual-cure material). After the first 8 weeks, bone healing was the most intense in controls, but after 12 weeks, the intensity of the healing process was similar in all groups, indicating the presence of osteogenesis for all the 3 endodontic sealers. Further research is necessary in order to assess long-term tissue response to these endodontic materials, since age and aging were not taken into consideration.

\section{REFERENCES}

1. Batista RF, Hidalgo MM, Hernandes L, Consolaro A, Velloso TR, Cuman RK (2007) Microscopic analysis of subcutaneous reactions to endodontic sealer implants in rats. J Biomed Mater Res A, 81: 171-177. doi: 10.1002/jbm.a.30918.

2. Bouillaguet S, Wataha JC, Tay FR, Brackett MG, Lockwood PE (2006) Initial in vitro biological response to contemporary 
endodontic sealers. J Endod, 32: 989-992. doi: 10.1016/j. joen.2006.05.006.

3. Develioglu H, Unver Saraydin S, Kartal U (2009) The bone-healing effect of a xenograft in a rat calvarial defect model. Dent Mater J, 28: 396-400. doi: 10.4012/dmj.28.396.

4. Farhad AR, Hasheminia S, Razavi S, Feizi M (2011) Histopathologic evaluation of subcutaneous tissue response to three endodontic sealers in rats. J Oral Science, 53: 15-21. doi: 10.2334/josnusd.53.15.

5. Gopikrishna V, Suresh Chandra B (2014) Grossman's endodontic practice. 13th ed. LWW.

6. Jie Zhao, Yu Liu, Wei-bin Sun, Hai Zhang (2011) Amorphous calcium phosphate and its application in dentistry. Chem Cent J, 5: 35-40. doi: 10.1186/1752-153X-5-40.

7. Kikuchi M (2013) Hydroxyapatite/collagen bone-like nanocomposite. Biol Pharm Bull, 36: 1666-1669. doi: 10.1248/ bpb.b13-00460.

8. Lodiene G, Morisbak E, Bruzell E, Orstavik D (2008) Toxicity evaluation of root canal sealers in vitro. IntEndod J, 41: 72-77. doi: 10.1111/j.1365-2591.2007.01321.x.

9. McNamara RP, Henry MA, Schindler WG, Hargreaves KM (2010) Biocompatibility of accelerated mineral trioxide aggregate in a rat model, J Endod, 36: 1851-1855. doi: 10.1016/j.joen.2010.08.021.

10. Moretton TR, Brown CE, Legan JJ, Kafrawy AH (2000) Tissue reactions after subcutaneous and intraosseous implantation of mineral trioxide aggregate and ethoxybenzoic acid cement. J Biomed Mater Res, 52: 528-533. doi: 10.1002/1097-4636(20001205)52:3<528::AID-JBM11>3.0.CO;2-9.

11. Olsson B, Sliwkowski A, Langeland K (1981) Subcutaneous implantation for the biological evaluation of endodontic materials. J Endod, 7: 355-336. doi: 10.1016/S00992399(81)80003-9.

12. Onay EO, Ungor M, Ozdemir BH (2007) In vivo evaluation of the biocompatibility of a new resin-based obturation system, Oral Surg Oral Med Oral Pathol Oral Radiol Endod, 104: e60-66. doi: 10.1016/j.tripleo.2007.03.006.

13. Porto GG, Vasconcelos BC, Andrade ES, Carneiro SC, Frota MS (2012) Is a $5 \mathrm{~mm}$ rat calvarium defect really critical? Acta Cir Bras, 27: 757-760. doi: 10.1590/S010286502012001100003.

14. Schmalz G., Arenholt-Bindslev D (2009) Biocompatibility of dental materials. Springer-Verlag, Berlin Heidelberg.

15. Sousa CJ, Montes CR, Pascon EA, Loyola AM, Versiani MA (2006) Comparison of the intraosseous biocompatibility of AH Plus, EndoREZ, and Epiphany root canal sealers. J Endod, 32: 656-662. doi: 10.1016/j.joen.2005.12.003.

16. Tienen TG, Heijkants RG, de Groot JH, Schouten AJ, Pennings AJ, Veth RP, Buma P (2006) Meniscal replacement in dogs. Tissue regeneration in two different materials with similar properties. J Biomed Mater Res B Appl Biomater, 76: 389-396. doi: 10.1002/jbm.b.30406.

17. Wataha JC, Rueggeberg FA, Lapp CA (1999) In vitro cytotoxicity of resin-containing restorative materials after aging in artificial saliva. Clin Oral Investigat, 3: 144-149. doi: 10.1007/s007840050093. 